\title{
Kinetics of charge inversion
}

\author{
Yan Levin and Jeferson J Arenzon \\ Instituto de Física, Universidade Federal do Rio Grande do Sul, Caixa Postal 15051, CEP \\ 91501-970, Porto Alegre, RS, Brazil \\ E-mail: levin@if.ufrgs.br
}

Received 10 October 2002, in final form 14 January 2003

Published 22 May 2003

Online at stacks.iop.org/JPhysA/36/5857

\begin{abstract}
Colloidal suspensions and polyelectrolyte solutions containing multivalent counterions can exhibit some very counter-intuitive behaviour usually associated with low-temperature physics. There are two particularly striking phenomena resulting from strong electrostatic correlations. One is the like-charge attraction and the second is the polyion overcharging. In this contribution we will concentrate on the problem of overcharging. In particular, we will explore the kinetic limitation to colloidal charge inversion in suspensions containing multivalent counterions.
\end{abstract}

PACS numbers: 41.20.Cv, 82.70.Dd, 82.35.Rs

\section{Introduction}

Colloidal suspensions and polyelectrolyte solutions containing multivalent counterions can exhibit some very curious electrostatic behaviour [1]. It is found that under some circumstances two like-charged polyions inside a suspension can actually attract one another [2-16]. The counterion-mediated attraction is responsible for DNA compaction inside bacteriophages, viruses that infect bacteria [17, 18], and for the organization of the eukaryotic cytoskeleton [19]. Another 'strange' electrostatic behaviour which can occur in suspensions containing multivalent counterions is the reversal of the electrophoretic mobility [1, 20-25]. The first thing that is learned in a course on electrostatics is that the force produced by the electric field on a charged particle is

$$
\mathbf{F}=Q \mathbf{E} .
$$

Thus, a positively charged particle, $Q>0$, is expected to move in the direction of the applied field while a negatively charged particle, $Q<0$, will move in the direction opposite to the field. This simple picture, however, breaks down inside a colloidal suspension of low dielectric solvent or even in aqueous suspensions containing multivalent counterions. The reason for the violation of the 'simple' physics learned in high school is the strong electrostatic 
many-body interactions between the colloidal particles and the counterions. The reversal of the electrophoretic mobility can be understood as a combination of two electrostatically driven mechanisms. Strong electrostatic interaction between colloids and counterions leads to the formation of polyion-counterion complexes [26-28]. The existence of counterion condensation has been known for over 30 years [29-31]; the general phenomenon is, however, much older than this and can be traced to the pioneering work of Bjerrum on ionic association inside electrolyte solutions almost 80 years ago [32]. In aqueous suspensions with only monovalent counterions, the net charge of complexes is of the same sign as the bare charge of the polyions.

If the solvent is water and the counterions are monovalent, the electrostatic interactions between the condensed counterions can be neglected [1], and the simplest Poisson-Boltzmann theory is sufficient to describe the polyion-counterion complexation [26, 33, 34]. In aqueous suspensions containing multivalent counterions or in suspension of low dielectric solvents, the electrostatic energy between the condensed counterions is significantly larger than the thermal energy and the electrostatic correlations between the condensed counterions can no longer be neglected. These electrostatic correlations can lead to colloidal overcharging, i.e. the net charge of the complex is of opposite sign to the charge of the bare polyion. The overcharged colloid will then move in the 'wrong' direction with respect to the applied electric field $[1,25]$.

\section{Overcharging}

To understand the phenomenon of overcharging we shall start by studying a very simple model. Consider a sphere of radius $a$ and fixed charge $-Z q$ distributed uniformly over its surface. We would like to know how many point-like $\alpha$-valent counterions, each of charge $\alpha q$, should be placed on top of this sphere in order to minimize the total electrostatic free energy [1, 22, 24, 35]. When we say 'counterions' we have in mind both simple multivalent ions such as $\mathrm{Ca}^{2+}$, as well as more complicated micelle-like aggregates with $\alpha$ significantly higher than one.

The free energy of a complex can be written as

$$
E_{n}=\frac{Z^{2} q^{2}}{2 \epsilon a}-\frac{Z \alpha n q^{2}}{\epsilon a}+F_{n}^{\alpha \alpha} .
$$

The first term is the self-energy of the charged sphere, the second term is the electrostatic energy of interaction between the sphere and $n$ condensed $\alpha$-ions, and the last term is the electrostatic energy of repulsion between the condensed counterions. To calculate the free energy of repulsion, it is convenient to express $F_{n}^{\alpha \alpha}$ in terms of the free energy of a onecomponent plasma (OCP), $n \alpha$-ions on the surface of a sphere with a uniform neutralizing background, $F_{n}^{\mathrm{OCP}}$. The free energy of a spherical OCP can be written as

$$
F_{n}^{\mathrm{OCP}}=F_{n}^{\alpha \alpha}-\frac{\alpha^{2} n^{2} q^{2}}{\epsilon a}+\frac{\alpha^{2} n^{2} q^{2}}{2 \epsilon a} .
$$

Substituting equation (3) into equation (2), the electrostatic free energy of a polyion-counterion complex becomes

$$
E_{n}=\frac{(Z-\alpha n)^{2} q^{2}}{2 \epsilon a}+F_{n}^{\mathrm{OCP}}
$$

In the strong coupling limit, corresponding to multivalent counterions or solvents of low dielectric permittivity, the free energy of the OCP is well approximated by the free energy of the low-temperature phase corresponding to a triangular Wigner crystal,

$$
F_{n}^{\mathrm{OCP}}=-M \frac{\alpha^{2} q^{2} n^{3 / 2}}{2 \epsilon a}
$$


where $M$ is the Madelung constant. For weaker couplings, the expression for the $F_{n}^{\mathrm{OCP}}$ can be obtained from the fits to the Monte Carlo data [36]. For concreteness we shall use $M=1.106$, the value appropriate for a planar Wigner crystal [1].

The effective charge of a polyion-counterion complex, in units of $-q$, is

$$
Z_{\text {eff }}=Z-\alpha n \text {. }
$$

The optimum number of condensed counterions $n^{*}$ is determined from the minimization of the total electrostatic free energy. We find $[1,22,24]$

$$
Z_{\mathrm{eff}}^{*}=-\frac{1+\sqrt{1+4 \gamma^{2} Z}}{2 \gamma^{2}} \approx-\frac{\sqrt{Z}}{\gamma}
$$

where

$$
\gamma=\frac{4}{3 M \sqrt{\alpha}}
$$

The optimal charge of a polyion-counterion complex is of opposite sign to the bare colloidal charge, i.e. the complex is overcharged. Inside the colloidal suspension containing multivalent counterions or solvents of low dielectric permittivity, the electrophoretic mobility can, therefore, be reversed.

Some care, however, must be taken in extrapolating the results of this simple model to real systems. While we have treated the counterions as condensed on top of the sphere, this is clearly not the case for a real colloidal suspension. Instead, the associated counterions form a layer around a colloidal particle which can be some nanometres wide. The presence of a simple electrolyte also strongly affects the net charge of the polyion- $\alpha$-ion complex [1]. Furthermore, the complex formation is a kinetic phenomenon requiring a counterion to overcome an energy barrier in order to join the already overcharged complex.

\section{Kinetics of overcharging}

In the previous section we have found that the minimum of the total electrostatic free energy of a polyion- $\alpha$-ion complex corresponds to an overcharged state. However, for a counterion to join an already overcharged complex it must overcome an energy barrier. The waiting time for a thermal fluctuation of sufficient strength necessary to drive a counterion over an activation barrier scales exponentially with the height of the barrier. There is, therefore, a kinetic limitation to the degree of overcharging which can prevent a thermodynamically optimum state from being reached on an experimental timescale. To explore this further we have to construct an effective interaction potential between a complex and a counterion separated by distance $r$.

The work necessary to bring a counterion from infinity to join a complex containing $n$ $\alpha$-ions is

$$
W=\frac{\mathrm{d} E_{n}}{\mathrm{~d} n}
$$

We define the reduced electrostatic potential of a counterion on the surface of the complex as $\varphi(a)=\beta W$, where $\beta=1 / k_{B} T$. Differentiating equation (4) we find

$$
\varphi(a)=-\frac{(Z-\alpha n) \lambda_{B} \alpha}{a}-\frac{3 M \alpha^{2} \sqrt{n}}{4 a}
$$

where $\lambda_{B}=q^{2} / \epsilon k_{B} T$. The first term of equation (10) is the electrostatic energy of interaction between a uniform spherical charge and an $\alpha$-ion, while the second term is due to electrostatic correlations between the $\alpha$-ions. We note that the correlational contribution 
used in equation (10) is correct only in the limit of strong electrostatic interactions between the condensed counterions, i.e. when the entropic contribution to the total free energy can be neglected. A more accurate approximation would be to use the full expression for the electrostatic free energy of the two-dimensional OCP obtained from the Monte Carlo simulations [36]. This, however, presents an unnecessary complication at the level of our semi-quantitative discussion. In addition, for weaker couplings, the simplified picture of a quasi-two-dimensional sheath of condensed counterions will probably break down, requiring a more careful analysis of the double layer. We will, therefore, restrict our analysis to the strong coupling limit. Under these conditions the correlational contribution to the interaction potential decays exponentially fast with the separation from the polyion surface [7, 15, 37]. The characteristic length is set by the average separation between the condensed counterions. More specifically, we can approximate the reduced interaction potential by

$$
\varphi(r)=-\frac{(Z-\alpha n) \lambda_{B} \alpha}{r}-\frac{3 M \alpha^{2} \sqrt{n}}{4 a} \mathrm{e}^{-(r-a) / \xi} .
$$

The decay of the correlational contribution is governed by the characteristic length $\xi$ which in the strong coupling limit is well approximated by [7, 15, 37]

$$
\xi=\frac{1}{|\mathbf{G}|}
$$

where $\mathbf{G}$ is the reciprocal lattice vector of a triangular Wigner crystal of condensed counterions. Due to strong coupling between the condensed counterions, equation (12) should remain a good approximation even significantly above the crystallization temperature. For a triangular Wigner crystal,

$$
|\mathbf{G}|=\frac{4 \pi}{\sqrt{3} b}
$$

where $b$ is the lattice spacing

$$
b=\frac{1}{3^{1 / 4} \sqrt{\sigma}}
$$

and $\sigma=n / 4 \pi a^{2}$ is the surface density of condensed counterions. Substituting equations (13) and (14) into equation (12), the decay length is found to be

$$
\xi=\frac{3^{1 / 4}}{2 \sqrt{\pi}} \frac{a}{\sqrt{n}} .
$$

For $n<Z / \alpha$, the electrostatic potential between a counterion and a complex is purely attractive favouring further counterion condensation. Inside an electrolyte solution this tendency towards polyion-counterion association is opposed by the loss of entropy resulting from the confinement of condensed counterions near the colloidal surface. Here, however, we shall not be concerned with the role of entropy [1].

For $n>Z / \alpha$ the interaction potential has two minima, one located at $r=a$ and the second at $r=\infty$. For $Z / \alpha<n<n^{*}$ the $r=a$ minimum is the dominant one, while for $n>n^{*}$ the global minimum changes to $r=\infty$. The value of $n^{*}$ corresponds to the number of condensed counterions which minimize the electrostatic free energy of the complex equation (4),

$$
n^{*}=\frac{Z-Z_{\mathrm{eff}}^{*}}{\alpha}
$$

In the case of trivalent counterions the energy barrier that a counterion needs to overcome in order to join a complex which already contains $n^{*}$ condensed $\alpha$-ions is less than $2 k_{B} T$ 


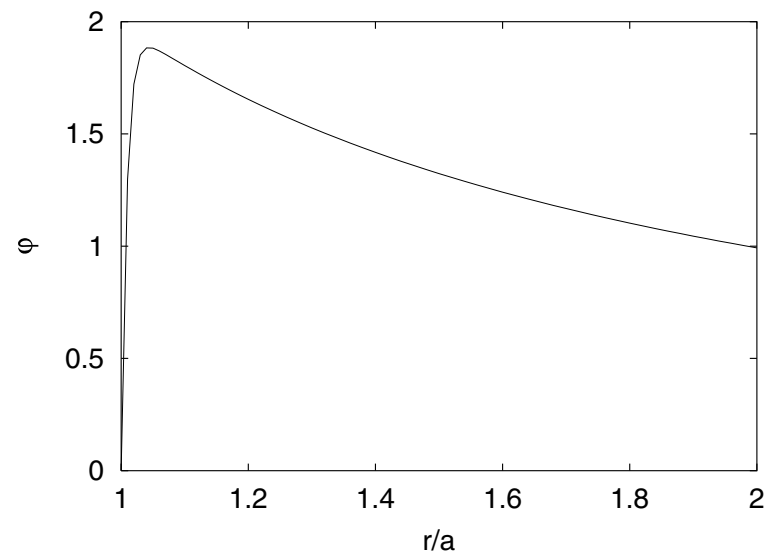

Figure 1. The reduced interaction potential between a complex with $Z=4000, a=1000 \AA, n=$ $n^{*}$ — given by equations (13) — condensed trivalent counterions, and a trivalent counterion located at distance $r$ from the centre of colloid.

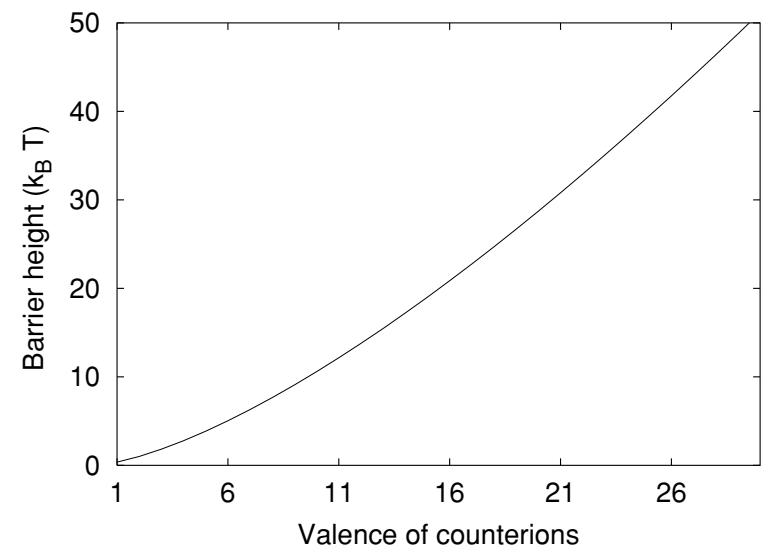

Figure 2. The height of the activation barrier that an $\alpha$-ion must overcome to join an optimally overcharged complex composed of a colloid with $Z=4000, a=1000 \AA$ and $n=n^{*}$ condensed $\alpha$-ions.

(figure 1). Thus, for trivalent counterions there is no kinetic hindrance to reaching the optimum overcharged state.

We next look at the height of the activation barrier as a function of the counterion valence (figure 2). It is clear that the height of the activation barrier grows rapidly with the increased valence of the $\alpha$-ions. In particular, we see that for $\alpha=10$ the activation barrier is already some $10 k_{B} T$ which is probably the maximum height that a counterion can overcome on a reasonable experimental timescale. Thus, the process of overcharging by the $\alpha$-ions with $\alpha>10$ will be kinetically controlled. For example, from equation (7) we see that the optimal state of overcharging of a colloidal particle of $Z=4000$ and radius $a=1000 \AA$ by micelles with $\alpha=25$ corresponds to $Z_{\text {eff }}^{*}=-271$. In practice, though, the process of overcharging will come to a stop when the barrier height reaches about $10 k_{B} T$, implying that the complex will stop growing when the net charge is only $Z_{\text {eff }}=-70$. 


\section{Conclusion}

In this contribution we have explored the kinetic limitation to overcharging. We find that kinetics does not play an important role for overcharging by simple multivalent counterions, so that the state of optimal overcharging, equation (7), is accessible within an experimental timescale. On the other hand, we find that the activation barrier grows rapidly with the valence of counterions, suggesting that the extent of overcharging by micelle-like aggregates is largely kinetically controlled.

The kinetic limitation to overcharging might also be important for the formation of DNAcationic lipid complexes. The problem of a reliable and safe mechanism for gene delivery is particularly pressing in view of the current medical applications. Strong electrostatic repulsion between DNA and a cellular membrane inhibits transfection of naked DNA into a cell. A way to overcome this difficulty is through the formation of overcharged complexes between DNA and cationic liposomes [38-42]. These lipoplexes having a net positive charge are attracted to the cellular membrane, facilitating the genetic transfection.

The presence of a simple electrolyte will also have a strong influence on the overcharging. It has been demonstrated that for sufficient concentration of $\alpha$-ions, monovalent salt favours overcharging $[1,43]$. In fact, in the presence of a simple electrolyte the thermodynamic state of optimum overcharging corresponds to the charge inversion of as much as $100 \%$. This should be contrasted with the result of equation (7), which shows that in the absence of salt, the effective charge of a complex scales as the square root of the bare charge. Salt will also lower the height of the activation barrier reducing the kinetic hindrance to overcharging.

The thermal fluctuations inside a colloidal suspension can result in a state in which some polyions are undercharged [44]. Although, the global free energy minimum corresponds to all colloids being overcharged, the overcharged-undercharged configurations are metastable and can lead to long-range attractions and colloidal phase separation. It should be very interesting to measure the life times of these metastabel states inside bulk suspensions. This should be possible to do using the Monte Carlo simulations [45].

Finally, near the isoelectric point where the net colloidal charge is close to zero, the van der Waals interactions become once again very important. The kinetics of macroion coagulation induced by the multivalent counterions has recently been studied in [46].

\section{References}

[1] Levin Y 2002 Rep. Prog. Phys. 651577

[2] Patey G N 1980 J. Chem. Phys. 725763

[3] Guldbrand L, Jonsson B, Wennerstrom H and Linse P 1984 J. Chem. Phys. 802221

[4] Kjellander R and Marcelja S 1986 J. Phys. Chem. 901230

[5] Stevens M J and Robbins M O 1990 Europhys. Lett. 1281

[6] Crocker J M and Grier D G 1994 Phys. Rev. Lett. 73352

[7] Rouzina I and Bloomfield V 1996 J. Chem. Phys. 1009977

[8] Ha B-Y and Liu A J 1997 Phys. Rev. Lett. 791289

[9] Levin Y, Arenzon J J and Stilck J F 1999 Phys. Rev. Lett. 832680

[10] Grønbech-Jensen N, Mashl R J, Bruinsma R F and Gelbart W M 1997 Phys. Rev. Lett. 782477

[11] Kornyshev A A and Leikin S 1999 Phys. Rev. Lett. 824138

[12] Allahyarov E, D’Amico I and Lowen H 1998 Phys. Rev. Lett. 811334

[13] Arenzon J J, Stilck J F and Levin Y 1999 Eur. Phys. J. B 1279

[14] Goulding D and Hansen J P 1999 Europhys. Lett. 46407

[15] Lau A W C, Pincus P, Levine D and Fertig H A 2001 Phys. Rev. E 63051604

[16] Diehl A, Carmona H A and Levin Y 2001 Phys. Rev. E 64011804

[17] Bloomfield V A 1991 Biopolymers 311471

[18] Bloomfield V A 1997 Biopolymer 44269 
[19] Tang J X and Janmey P A 1996 J. Biol. Chem. 2718556

[20] Lozada-Cassou M, Saavedra-Barrera R and Henderson D 1982 J. Chem. Phys. 775150

[21] Gonzales-Tovar E, Lozada-Cassou M and Henderson D 1985 J. Chem. Phys. 83361

[22] Shklovskii B I 1999 Phys. Rev. E 605802

[23] Nguyen T T, Grosberg A Y and Shklovskii B I 2000 Phys. Rev. Lett. 851568

[24] Messina R, Holm C and Kremer K 2001 Phys. Rev. E 64021405

[25] Grosberg A Y, Nguyen T T and Shklovskii B I 2002 Rev. Mod. Phys. 74329

[26] Alexander S et al 1984 J. Chem. Phys. 805776

[27] Levin Y, Barbosa M C and Tamashiro M N 1998 Europhys. Lett. 41123

[28] Diehl A, Barbosa M C and Levin Y 2001 Europhys. Lett. 5386

[29] Manning G S 1969 J. Chem. Phys. 51924

[30] Manning G S 1978 Q. Rev. Biophys. II 2179

[31] Oosawa F 1971 Polyelectrolytes (New York: Dekker)

[32] Bjerrum N 1926 Kgl. Dansk. Vidensk. Selsk. Mat.-Fys. Meddr. 71

[33] Trizac E, Bocquet L and Aubouy M 2002 Phys. Rev. Lett. 89248301

[34] Bocquet L, Trizac E and Aubouy M 2002 J. Chem. Phys. 1178138

[35] Thomson J J 1904 Phil. Mag. 7237

[36] Gann R C, Chakravarty S and Chester G V 1979 Phys. Rev. B 20326

[37] Stilck J F, Levin Y and Arenzon J J 2002 J. Stat. Phys. 106287

[38] Felgner P L and Ringold G M 1989 Nature 337387

[39] Felgner P L 1987 Sci. Am. 27686

[40] Friedmann T 1997 Sci. Am. 27680

[41] Hope M J, Mui B, Ansell S and Ahkong Q F 1998 Mol. Membrane Biol. 151

[42] Kuhn P S, Levin Y and Barbosa M C 1999 Physica A 2748

[43] Nguyen T T, Grosberg A Y and Shklovskii B I 2000 Phys. Rev. Lett. 851568

[44] Messina R, Holm C and Kremer K 2000 Phys. Rev. Lett. 85872

[45] Linse P and Lobaskin V 2000 J. Chem. Phys. 1123917

[46] Nguyen T T and Shklovskii B I 2002 Phys. Rev. E 65031409 